\title{
Description du premier Campodéidé cavernicole du Guatemala
}

\author{
par \\ B. CONDÉ
}

Avec 2 figures

\section{SUMMARY}

Juxtlacampa hauseri n. sp. is described from the Cueva Chirrepeck in Guatemala. Its affinities with the other species of this genus, juxtlahuacensis, are discussed.

Les grottes du Mexique hébergent d'intéressants Campodéidés, étudiés par P. WYGODZINSKY (1944), parmi lesquels on peut reconnaître des espèces voisines de formes endogées [Campodea chica, Podocampa cavernicola (sub Parallocampa), Litocampa atoyacensis (sub Plusiocampa)], tandis que d'autres ont nécessité la création de deux genres nouveaux, jusqu'ici monospécifiques: Paratachycampa boneti du Nuevo Léon et Juxtlacampa juxtlahuacensis du Guerrero. Ces dernières espèces ont des antennes relativement longues (de 27 à plus de 35 articles) et leurs cerques surpassent la longueur du corps (trois fois chez Juxtlacampa), caractères fréquents chez les cavernicoles.

On pouvait logiquement s'attendre à rencontrer des représentants des mêmes lignées dans les grottes du Guatemala, explorées par P. Strinati en 1973. Les Campodéidés y sont certainement fort peu abondants, puisqu'un unique spécimen juvénile figure parmi les récoltes de ce chercheur expérimenté. Il m'a été soumis par le Dr. B. Hauser, auquel je dédie amicalement la seconde espèce connue de Juxtlacampa.

\section{Juxtlacampa hauseri n. sp.}

Guatemala, Alta Verapaz, Cueva Chirrepeck, 6 ou 8 avril 1973: 1 f juv. (P. Strinati). Holotype monté in toto dans le médium II de Marc André. Muséum d'Histoire naturelle de Genève, Département des Arthropodes.

Longueurs. - Corps: 2,5 mm; cerque (extrémité distale brisée) : 4,5 mm.

Téguments. - Epicuticule sans ornementations; soies de revêtement glabres.

Tête. - Antennes de 19 articles à peine plus longs que larges. Sensille du III ${ }^{\mathrm{C}}$ article latéro-sternal, entre $d$ (très peu différencié) et $e$, subcylindrique, assez allongé. 
Article apical environ 2 fois $1 / 2$ aussi long que large; l'organe cupuliforme, assez volumineux, renferme 8 sensilles à l'antenne gauche et 10 à l'antenne droite; leur forme est intermédiaire entre ceux des formes endogées et ceux des espèces cavernicoles évoluées (digitations peu nombreuses et courtes). Sensille du palpe maxillaire très légèrement fusiforme; sensille latéro-externe du palpe labial un peu arqué.

Processus frontal $(=$ rostre) bien individualisé, sa portion apicale plus sclérifiée; il porte un seul macrochète glabre et une demie douzaine de soies. $3+3$ macrochètes bordent la ligne d'insertion des antennes, l'antérieur $(a=41)$ et l'intermédiaire $(i=45)$ avec 3 ou 4 barbules, le postérieur $(p=34)$ glabre. Phanères $x$ non différenciés.

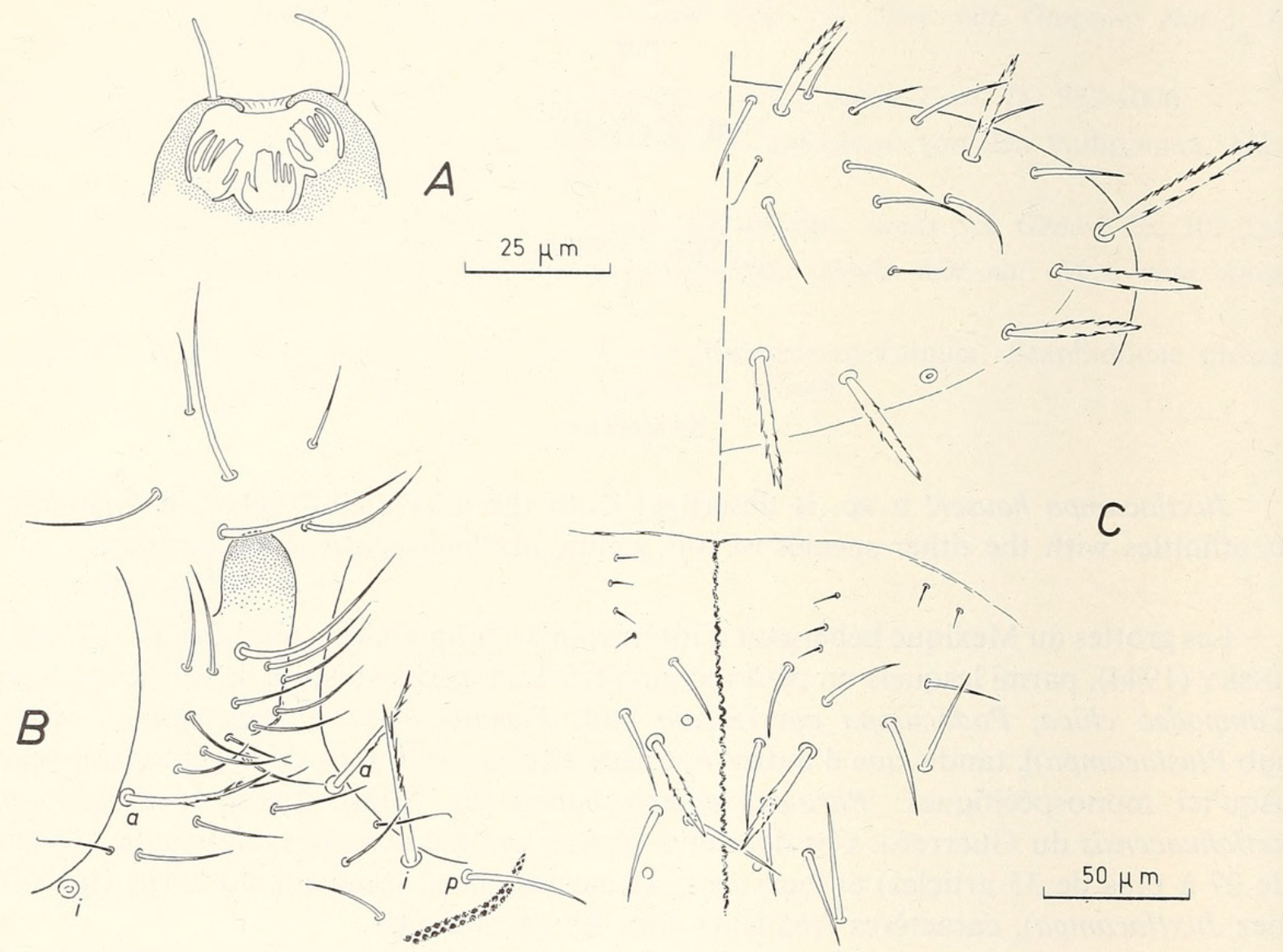

FIG. 1.

Juxtlacampa hauseri n. sp., holotype.

$A$. Organe apical en coupe optique. - B. Processus frontal. -

$C$. Pronotum et portion antérieure du mésonotum.

Explication des lettres dans le texte.

Thorax.-Répartition des macrochètes:

$\begin{array}{cccc} & \text { ma } & \text { la } & l p \\ \text { Th. I } & 1+1 & 1+1 & 1+1 \\ \text { Th. II } & 1+1 & 0 & 0\end{array}$

Cette formule réduite, ne comportant pas de macrochètes métanotaux, est aussi celle de l'espèce-type du genre et de certains Metriocampa s. str. Les machrochètes sont courts et relativement épais, de forme analogue à celle des soies marginales. Les ma et la 
du pronotum, et les $m a$ du mésonotum sont sensiblement égaux entre eux (29-33), les $l p$ pronotaux étant 1 fois $2 / 3$ aussi longs (50).

Fémur III sans macrochète tergal, mais un court et grêle macrochète fourchu est présent à la face sternale du tibia, vers la moitié de sa longueur (non mentionné chez le type). Les 3 longues soies subapicales du tarse sont entièrement glabres, mais les soies de la paire sternale la plus distale et une soie latérale postérieure voisine sont densément et régulièrement barbelées sur toute leur longueur. Griffes subégales à talon marqué, ne recouvrant pas l'apex du tarse; les crêtes latérales sont de dimensions réduites, mais ornées de fines stries obliques; processus télotarsaux sétiformes, glabres ,progressivement atténués, sensiblement de la longueur des griffes.

Abdomen. - Tergites I à VII sans macrochètes; tergite VIII avec une seule paire de latéraux postérieurs; segment IX avec, au total, $5+5$ macrochètes, dont $3+3$ appartiennent au tergite. Valvule supra-anale portant 5 soies dont une subapicale médiane.

Sternite I avec $7+7$ macrochètes bien différenciés, répartis de façon typique ( $4+4$ sur la région médiane de la plaque sternale et 3 sur chaque portion latérale); appendices subcylindriques avec 4 poils $a_{1}$ seulement; sternites II à VII avec $6+6$ macrochètes bien différenciés et $2+2$ parastylaires faibles; styles banaux, leurs phanères moins barbelés que chez l'espèce-type: moyenne sternale fourchue, subapicale avec une ou deux branches, apicale avec 2 branches basilaires bien développées, surtout la plus distale; sternite VIII avec $3+3$.

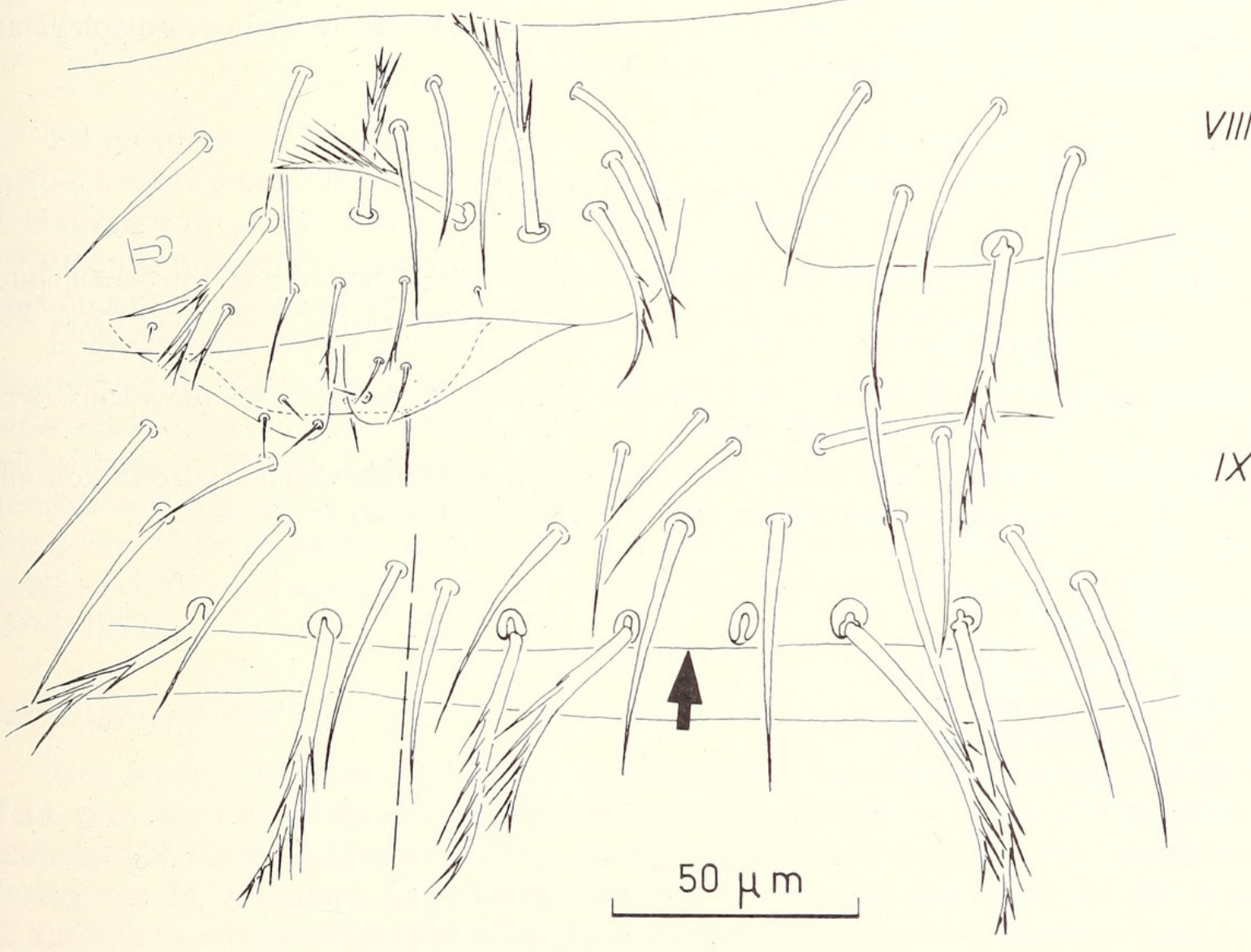

FIG. 2.

Juxtlacampa hauseri $\mathrm{n}$. sp., holotype.

Sternites et tergites VIII et IX.

La flèche indique la limite des territoires sternal et tergal du segment IX. 
Papille génitale avec 2 soies asymétriques (une courte dorsale, une longue subapicale) sur le tubercule et 3 poils, insérés en triangle, sur chaque volet.

Cerques à base longue et mal limitée, suivie d'environ 23 articles; leur revêtement est court, mais tous les articles portent des verticilles (généralement deux) de macrochètes au moins fourchus avec, vers la base du cerque, une $3^{\mathrm{e}}$ branche, plus petite, sur la branche latérale de la fourche.

Affinités. - L'appartenance de la nouvelle espèce au genre Juxtlacampa n'est pas douteuse. La réduction du nombre des macrochètes sur les tergites abdominaux et, plus encore, sur le méso- et le métanotum constituent des ressemblances morphologiques suffisantes, et le voisinage géographique des deux espèces plaide en faveur d'une parenté réelle, plutôt que d'une hypothétique convergence. La forme des processus télotarsaux, laminés et pileux chez le type, mais sétiformes chez notre espèce, ne peut plus actuellement constituer une divergence d'ordre générique, comme je l'ai souligné à plusieurs reprises (en particulier 1962, p. 93), les particularités de ces organites étant même, dans certains cas, des critères spécifiques inconstants (1959).

Plusieurs autres caractères séparent hauseri de juxtlahuacensis. Les antennes sont beaucoup plus courtes, leurs articles étant moins nombreux (19, au lieu de 27-30) et moins allongés; un macrochète est présent sur la face sternale du tibia III; il n'y a qu'une seule paire de macrochètes au tergite VIII (au lieu de $2+2$ ); enfin, les sternites I et II à VII portent un nombre typique de macrochètes $(7+7$ et $6+6)$, alors qu'il existe une néotrichie importante $(15+15$ ou 14 et $7-9+7-9)$, comportant des dispositions asymétriques, chez juxtlahuacensis. Les deux espèces semblent représenter deux paliers évolutifs d'une même lignée, des caractères fréquents chez les formes cavernicoles étant plus accentués chez l'espèce-type que chez hauseri.

\section{BIBLIOGRAPHIE}

Condé, B. 1959. Métamorphose des processus télotarsaux d'un Campodéidé (Insecte Diploure) au cours d'une mue postpubérale. C. r. Séanc. hebd. Acad. Sci., Paris, 248: $1402-1405$.

Condé, B. et Geeraert, P. Melle, 1962. Campodéidés endogés du centre des Etats-Unis. Archs Zool. exp. gén. 101: 73-160.

Wygodzinsky, P. 1944. Contribuiçao ao conhecimento da familia Campodeidae (Entotrophi, Insecta) do Mexico. An. Esc. nac. Cienc. biol. Méx. 3: 367-404.

Adresse de l'auteur :

Laboratcire de Zoologie

Université de Nancy I

34 , rue Sainte-Catherine

F-54 Nancy

France 


\section{$2 \mathrm{BHL}$ Biodiversity Heritage Library}

1975. "Description du premier campodeide cavernicole du Guatemala." Revue suisse de zoologie 82, 421-424. https://doi.org/10.5962/bhl.part.78266.

View This Item Online: https://www.biodiversitylibrary.org/item/127363

DOI: https://doi.org/10.5962/bhl.part.78266

Permalink: https://www.biodiversitylibrary.org/partpdf/78266

\section{Holding Institution}

Smithsonian Libraries

\section{Sponsored by}

Biodiversity Heritage Library

\section{Copyright \& Reuse}

Copyright Status: In Copyright. Digitized with the permission of the rights holder.

Rights Holder: Muséum d'histoire naturelle - Ville de Genève License: http://creativecommons.org/licenses/by-nc-sa/3.0/

Rights: https://www.biodiversitylibrary.org/permissions/

This document was created from content at the Biodiversity Heritage Library, the world's largest open access digital library for biodiversity literature and archives. Visit BHL at https://www.biodiversitylibrary.org. 